\title{
The NESTOR Framework: How to Handle Hierarchical Data Structures
}

\author{
Nicola Ferro and Gianmaria Silvello \\ Department of Information Engineering, University of Padua, Italy \\ \{ferro, silvello\}@dei.unipd.it
}

\begin{abstract}
In this paper we study the problem of representing, managing and exchanging hierarchically structured data in the context of a Digital Library (DL). We present the NEsted SeTs for Object hieRarchies (NESTOR) framework defining two set data models that we call: the "Nested Set Model (NS-M)" and the "Inverse Nested Set Model (INS$\mathrm{M})$ " based on the organization of nested sets which enable the representation of hierarchical data structures. We present the mapping between the tree data structure to NS-M and to INS-M. Furthermore, we shall show how these set data models can be used in conjunction with Open Archives Initiative Protocol for Metadata Harvesting (OAI-PMH) adding new functionalities to the protocol without any change to its basic functioning. At the end we shall present how the couple OAI-PMH and the set data models can be used to represent and exchange archival metadata in a distributed environment.
\end{abstract}

\section{Motivations}

In Digital Library Systems (DLSs) objects are often organized in hierarchies to help in representing, managing or browsing them. For instance, books in a library can be classified by author and then by subject and then by publishing house. Documents in an archive are organized in a hierarchy divided into fonds, subfonds, series, sub-series and so on. In the same way the internal structure of an object can be hierarchical; for example the structure of a book organized in chapters, sections and subsections or a web page composed by nested elements such as body, titles, subtitles, paragraphs and subparagraphs. One very important tool extensively adopted to represent digital objects such as metadata, text documents and multimedia contents - the eXtensible Markup Language (XML) - has an intrinsically hierarchical structure.

Representing, managing, preserving and sharing efficiently and effectively the hierarchical structures is a key point for the development and the consolidation of DLS technology and services. In this paper we propose the NEsted SeTs for Object hieRarchies (NESTOR 1 framework defining two set data models that we call: the "Nested Set Model (NS-M)" and the "Inverse Nested Set Model (INSM)". These models are defined in the context of the ZFC (Zermelo-Fraenkel with

\footnotetext{
${ }^{1}$ Nestor is a Greek myth [1; a king of Pylos in Peloponnesus, who in old age led his subjects to the Trojan War. His wisdom and eloquence were proverbial.
} 
the axiom of Choice) axiomatic set theory [7, exploiting the advantages of the use of sets in place of a tree structure. The foundational idea behind these set data models is that an opportune set organization can maintain all the features of a tree data structure with the addition of some new relevant functionalities. We define these functionalities in terms of flexibility of the model, rapid selection and isolation of easily specified subsets of data and extraction of only those data necessary to satisfy specific needs.

Furthermore, these set data models can work in conjunction with the Open Archives Initiative Protocol for Metadata Harvesting (OAI-PMH) [12 that is the standard de-facto for metadata sharing between DLSs in distributed environments. The extension of OAI-PMH with these set data models allows the protocol to manage and exchange complex hierarchical data structure in a flexible way. The extension of OAI-PMH shall permit the exchange of data belonging to a hierarchy with a variable granularity without losing the relationships with the other data in the hierarchy. Furthermore, the OAI-set which is a constituent part of the protocol will be used also to organize the data and not only to enable the selective harvesting. A concrete use case is the archival data that are organized in a hierarchy which preserve the meaningful relationships between the data. When an archival object is shared it has to preserve all the relationships with the preservation context and with the other objects in the archive; since the use of tree data structure in this context turns out to be problematic in terms of accessibility and flexibility, we shall show that the use of the proposed data models in conjunction with OAI-PMH overcomes many of these issues.

The paper is organized as follows: Section 2 briefly defines the tree data structure. Section 3 defines the two proposed set data models and presents the mapping functions between the tree data structure and the set data models. Section [4] describes how OAI-PMH can extend its functionalities by exploiting the NS-M or the INS-M; moreover this section presents a use case in which the set data models and OAI-PMH can be used together to exchange full expressive archival metadata. Section 5 draws some conclusions.

\section{The Tree Data Structure}

The most common and diffuse way to represent a hierarchy is the tree data structure, which is one of the most important non-linear data structures in computer science 8 . We define a tree as $T(V, E)$ where $V$ is the set of nodes and $E$ the set of edges connecting the nodes. $V$ is composed by $n$ nodes $V=\left\{v_{1}, \ldots, v_{n}\right\}$ and $E$ is composed by $n-1$ edges. If $v_{i}, v_{j} \in V$ and if $e_{i j} \in E$ then $e_{i j}$ is the edge connecting $v_{i}$ to $v_{j}$, thus $v_{i}$ is the parent of $v_{j}$. We indicate with $d_{V}^{-}\left(v_{i}\right)$ the inbound degree of node $v_{i} \in V$ representing the number of its inbound edges; $d_{V}^{+}\left(v_{i}\right)$ is the outbound degree of $v_{i} \in V$ representing the number of its outbound edges. $v_{r} \in V$ is defined to be the root of $T(V, E)$ if and only if $d_{V}^{-}\left(v_{r}\right)=0 ; \forall v_{i} \in V \backslash\left\{v_{r}\right\}, d_{V}^{-}\left(v_{i}\right)=1$. The set of all external nodes is $V_{e x t}=\left\{v_{i}: d_{V}^{+}\left(v_{i}\right)=0\right\}$ and the set of all the internal nodes is $V_{\text {int }}=\left\{v_{i}: d_{V}^{+}\left(v_{i}\right)>0\right\}$. 
We define with $\Gamma_{V}^{+}\left(v_{i}\right)$ the set of all the descendants of $v_{i}$ in $V$ (including $v_{i}$ ifself); vice versa $\Gamma_{V}^{-}\left(v_{i}\right)$ is the set of all the ancestors of $v_{i}$ in $V$ (including $v_{i}$ ifself). We shall use the set $\Gamma$ in the following of this work, so it is worth underlining a couple of recurrent cases. Let $v_{r} \in V$ be the root of a tree $T(V, E)$ then $\Gamma_{V}^{-}\left(v_{r}\right)=\left\{v_{r}\right\}$ and $\Gamma_{V}^{+}\left(v_{r}\right)=V$. Furthermore, let $v_{i}$ an external node of $T(V, E)$, then $\Gamma_{V}^{+}\left(v_{i}\right)=\left\{v_{i}\right\}$.

\section{The Set Data Models}

We propose two set data models called Nested Set Model (NS-M) and Inverse Nested Set Model (INS-M) based on an organization of nested sets. The most intuitive way to understand how these models work is to relate them to the well-know tree data structure. Thus, we informally present the two data models by means of examples of mapping between them and a sample tree.

The first model we present is the Nested Set Model (NS-M). The intuitive graphic representation of a tree as an organization of nested sets was used in [8] to show different ways to represent tree data structure and in 3 to explain an alternative way to solve recursive queries over trees in SQL language. An organization of sets in the NS-M is a collection of sets in which any pair of sets is either disjoint or one contains the other. In Figure 1 we can see how a sample tree is mapped into an organization of nested sets based on the NS-M.

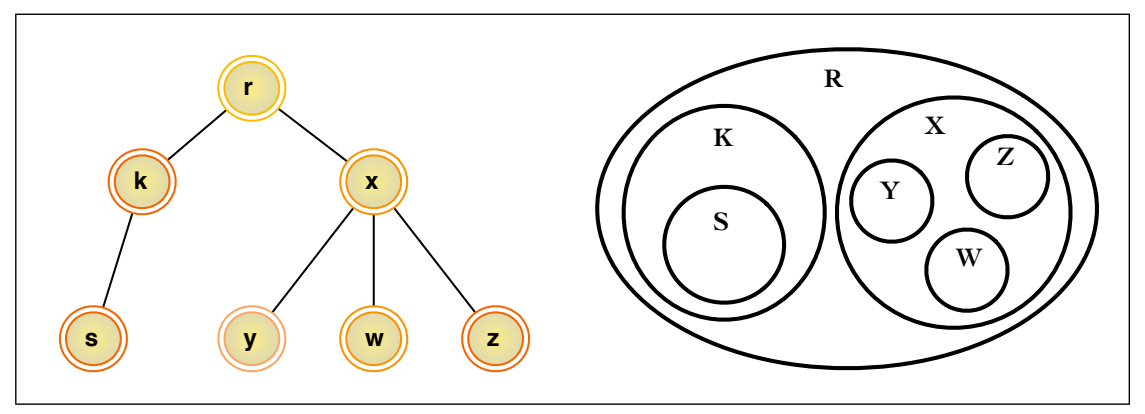

Fig. 1. The mapping between a tree data structure and the Nested Set Model

From Figure 1 we can see that each node of the tree is mapped into a set, where child nodes become proper subsets of the set created from the parent node. Every set is subset of at least of one set; the set corresponding to the tree root is the only set without any supersets and every set in the hierarchy is subset of the root set. The external nodes are sets with no subsets. The tree structure is maintained thanks to the nested organization and the relationships between the sets are expressed by the set inclusion order. Even the disjunction between two sets brings information; indeed, the disjunction of two sets means that these belong to two different branches of the same tree. 
The second data model is the Inverse Nested Set Model (INS-M). We can say that a tree is mapped into the INS-M transforming each node into a set, where each parent node becomes a subset of the sets created from its children. The set created from the tree's root is the only set with no subsets and the root set is a proper subset of all the sets in the hierarchy. The leaves are the sets with no supersets and they are sets containing all the sets created from the nodes composing tree path from a leaf to the root. An important aspect of INS-M is that the intersection of every couple of sets obtained from two nodes is always a set representing a node in the tree. The intersection of all the sets in the INS-M is the set mapped from the root of the tree. Fig. 2 shows the organization of nested sets created from the branch of a tree.

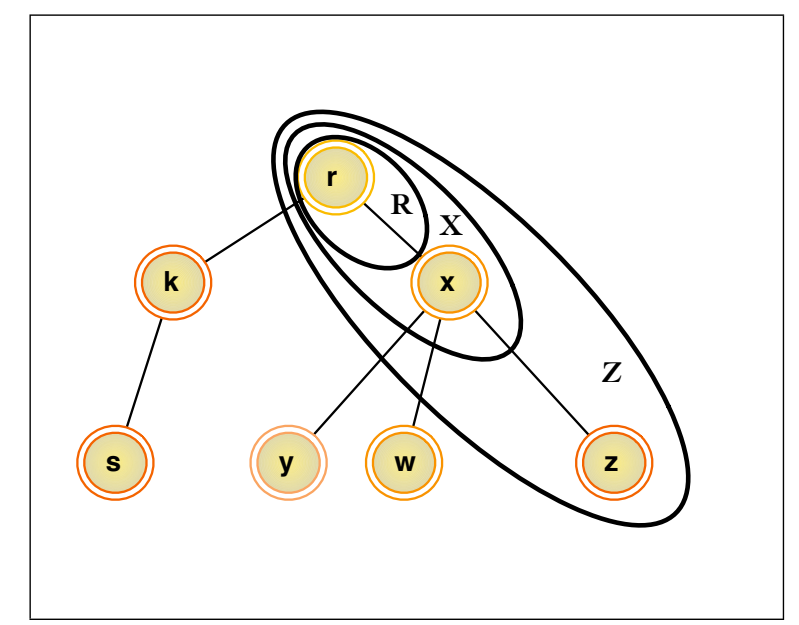

Fig. 2. How the branch of a tree can be mapped into the INS-Model

It is worthwhile for the rest of the work to define some basic concepts of set theory: the family of subsets and the subfamily of subsets, with reference to 4 for their treatment. However, we assume the reader is confident with the basic concepts of ZFC axiomatic set theory, which we cannot extensively treat here for space reasons.

Definition 1. Let $A$ be a set, $I$ a non-empty set and $\mathcal{C}$ a collection of subsets of $A$. Then a bijective function $\mathcal{A}: I \longrightarrow \mathcal{C}$ is a family of subsets of $A$. We call $I$ the index set and we say that the collection $\mathcal{C}$ is indexed by $I$.

We use the following notation $\left\{A_{i}\right\}_{i \in I}$ to indicate the family $\mathcal{A}$; the notation $A_{i} \in\left\{A_{i}\right\}_{i \in I}$ means that $\exists i \in I \mid \mathcal{A}(i)=A_{i}$. We call subfamily of $\left\{A_{i}\right\}_{i \in I}$ the restriction of $\mathcal{A}$ to $J \subseteq I$ and we denote this with $\left\{B_{i}\right\}_{j \in J} \subseteq\left\{A_{i}\right\}_{i \in I}$. 
Definition 2. Let $A$ be a set and let $\left\{A_{i}\right\}_{i \in I}$ be a family. Then $\left\{A_{i}\right\}_{i \in I}$ is a Nested Set family if:

$$
\begin{aligned}
& A \in\left\{A_{i}\right\}_{i \in I}, \\
& \emptyset \notin\left\{A_{i}\right\}_{i \in I}, \\
& \forall A_{h}, A_{k} \in\left\{A_{i}\right\}_{i \in I}, h \neq k \mid A_{h} \cap A_{k} \neq \emptyset \Rightarrow A_{h} \subset A_{k} \vee A_{k} \subset A_{h} .
\end{aligned}
$$

Thus, we define a Nested Set family (NS-F) as a family where three conditions must hold. The first condition (3.1) states that set $A$ which contains all the sets in the family must belong to the NS-F. The second condition states that the empty-set does not belong to the NS-F and the last condition (3.3) states that the intersection of every couple of distinct sets in the NS-F is not the empty-set only if one set is a proper subset of the other one 612.

Theorem 1. Let $T(V, E)$ be a tree and let $\Phi$ be a family where $I=V$ and $\forall v_{i} \in V, V_{v_{i}}=\Gamma_{V}^{+}\left(v_{i}\right)$. Then $\left\{V_{v_{i}}\right\}_{v_{i} \in V}$ is a Nested Set family.

Proof. Let $v_{r} \in V$ be the root of the tree then $V_{v_{r}}=\Gamma_{V}^{+}\left(v_{r}\right)=V$ and thus $V \in\left\{V_{v_{i}}\right\}_{v_{i}}$ (condition 3.1). By definition of descendant set of a node, $\forall v_{i} \in V$, $\left|V_{v_{i}}\right|=\left|\Gamma_{V}^{+}\left(v_{i}\right)\right| \geq 1$ and so $\emptyset \notin\left\{V_{v_{i}}\right\}_{v_{i} \in V}$ (condition 3.2).

Now, we prove condition 3.3. Let $v_{h}, v_{k} \in V, h \neq k$ such that $V_{v_{h}} \cap V_{v_{k}}=$ $\Gamma_{V}^{+}\left(v_{h}\right) \cap \Gamma_{V}^{+}\left(v_{k}\right) \neq \emptyset$, ab absurdo suppose that $\Gamma_{V}^{+}\left(v_{h}\right) \nsubseteq \Gamma_{V}^{+}\left(v_{k}\right) \wedge \Gamma_{V}^{+}\left(v_{k}\right) \nsubseteq$ $\Gamma_{V}^{+}\left(v_{k}\right)$. This means that the descendants of $v_{h}$ share at least a node with the descendants of $v_{k}$ but they do not belong to the same subtree. This means that $\exists v_{z} \in V \mid d_{V}^{-}\left(v_{z}\right)=2$ but then $T(V, E)$ is not a tree.

Example 1. Let $T(V, E)$ be a tree where $V=\left\{v_{0}, v_{1}, v_{2}, v_{3}\right\}$ and $E=\left\{e_{01}, e_{02}\right.$, $\left.e_{23}\right\}$, thus $\Gamma_{V}^{+}\left(v_{0}\right)=\left\{v_{0}, v_{1}, v_{2}, v_{3}\right\}, \Gamma_{V}^{+}\left(v_{1}\right)=\left\{v_{1}\right\}, \Gamma_{V}^{+}\left(v_{2}\right)=\left\{v_{2}, v_{3}\right\}$ and $\Gamma_{V}^{+}\left(v_{3}\right)=\left\{v_{3}\right\}$. Let $\left\{V_{v_{i}}\right\}_{v_{i} \in V}$ be a family, where $V_{v_{0}}=\left\{v_{0}, v_{1}, v_{2}, v_{3}\right\}, V_{v_{1}}=\left\{v_{1}\right\}$, $V_{v_{2}}=\left\{v_{2}, v_{3}\right\}$ and $V_{v_{3}}=\left\{v_{3}\right\}$. Then, from theorem 1 it follows that $\left\{V_{v_{i}}\right\}_{v_{i} \in V}$ is a NS-F.

In the same way we can define the Inverse Nested Set Model (INS-M):

Definition 3. Let $A$ be a set and let $\left\{A_{i}\right\}_{i \in I}$ be a family. Then $\left\{A_{i}\right\}_{i \in I}$ is an Inverse Nested Set family if:

$$
\begin{aligned}
& \emptyset \notin\left\{A_{i}\right\}_{i \in I}, \\
& \forall\left\{B_{j}\right\}_{j \in J} \subseteq\left\{A_{i}\right\}_{i \in I} \Rightarrow \bigcap_{j \in J} B_{j} \in\left\{A_{i}\right\}_{i \in I} .
\end{aligned}
$$

Thus, we define an Inverse Nested Set family (INS-F) as a family where two conditions must hold. The first condition (3.4) states that the empty-set does not belong to the INS-F. The second condition states that the intersection of every subfamily of the INS-F belongs to the INS-F itself.

Theorem 2. Let $T(V, E)$ be a tree and let $\Psi$ be a family where $I=V$ and $\forall v_{i} \in V, V_{v_{i}}=\Gamma_{V}^{-}\left(v_{i}\right)$. Then $\left\{V_{v_{i}}\right\}_{v_{i} \in V}$ is an Inverse Nested Set family. 
Proof. By definition of the set of the ancestors of a node, $\forall v_{i} \in V,\left|V_{v_{i}}\right|=$ $\left|\Gamma_{V}^{-}\left(v_{i}\right)\right| \geq 1$ and so $\emptyset \notin\left\{V_{v_{i}}\right\}_{v_{i} \in V}$ (condition 3.4).

Let $\left\{B_{v_{j}}\right\}_{v_{j} \in J}$ be a subfamily of $\left\{V_{v_{i}}\right\}_{v_{i} \in V}$. We prove condition 3.5 by induction on the cardinality of $J .|J|=1$ is the base case and it means that every subfamily $\left\{B_{v_{j}}\right\}_{v_{j} \in J} \subseteq\left\{V_{v_{i}}\right\}_{v_{i} \in V}$ is composed only by one set $B_{v_{1}}$ whose intersection is the set itself and belongs to the family $\left\{V_{v_{i}}\right\}_{v_{i} \in V}$ by definition.

For $|J|=n-1$ we assume that $\exists v_{n-1} \in V \mid \bigcap_{v_{j} \in J} B_{v_{j}}=B_{v_{n-1}} \in\left\{V_{v_{i}}\right\}_{v_{i} \in V}$; equivalently we can say that $\exists v_{n-1} \in V \mid \bigcap_{v_{j} \in J} \Gamma_{V}^{-}\left(v_{j}\right)=\Gamma_{V}^{-}\left(v_{n-1}\right)$, thus, $\Gamma_{V}^{-}\left(v_{n-1}\right)$ is a set of nodes that is composed of common ancestors of the $n-1$ considered nodes.

For $|J|=n$, we have to show that $\exists v_{t} \in V \mid \forall v_{n} \in J, B_{v_{n-1}} \cap B_{v_{n}}=B_{v_{t}} \in$ $\left\{V_{v_{i}}\right\}_{v_{i} \in V}$. This is equivalent to show that $\exists v_{t} \in V \mid \forall v_{n} \in J, \Gamma_{V}^{-}\left(v_{n-1}\right) \cap$ $\Gamma_{V}^{-}\left(v_{n}\right)=\Gamma_{V}^{-}\left(v_{t}\right)$.

Ab absurdo suppose that $\exists v_{n} \in J \mid \forall v_{t} \in V, \Gamma_{V}^{-}\left(v_{n-1}\right) \cap \Gamma_{V}^{-}\left(v_{n}\right) \neq \Gamma_{V}^{-}\left(v_{t}\right)$. This would mean that $v_{n}$ has no ancestors in $J$ and, consequently, in $V$; at the same time, this would mean that $v_{n}$ is an ancestor of no node in $J$ and, consequently, in $V$. But this means that $V$ is the set of nodes of a forest and not of a tree.

Example 2. Let $T(V, E)$ be a tree where $V=\left\{v_{0}, v_{1}, v_{2}, v_{3}\right\}$ and $E=\left\{e_{01}, e_{02}, e_{23}\right\}$, thus $\Gamma_{V}^{-}\left(v_{0}\right)=\left\{v_{0}\right\}, \Gamma_{V}^{-}\left(v_{1}\right)=\left\{v_{0}, v_{1}\right\}, \Gamma_{V}^{-}\left(v_{2}\right)=\left\{v_{0}, v_{2}\right\}$ and $\Gamma_{V}^{-}\left(v_{3}\right)=\left\{v_{0}, v_{2}, v_{3}\right\}$. Let $\left\{V_{v_{i}}\right\}_{v_{i} \in V}$ be a family where $V_{v_{0}}=\left\{v_{0}\right\}, V_{v_{1}}=$ $\left\{v_{0}, v_{1}\right\}, V_{v_{2}}=\left\{v_{1}, v_{2}\right\}$ and $V_{v_{3}}=\left\{v_{0}, v_{2}, v_{3}\right\}$. Then, from theorem 圆 it follows that $\left\{V_{v_{i}}\right\}_{v_{i} \in V}$ is a INS-F.

\section{Set-Theoretic Extensions of OAI-PMH}

The defined set data models can be exploited to improve the data exchange between DLSs in a distributed environment. In this context the standard defacto for metadata exchange between DLSs is the couple OAI-PMH and XML. The main reason is the flexibility of both the protocol and the XML that foster interoperability between DLSs managing different kinds of metadata coming from different kinds of cultural organizations. It is worthwhile to describe the functioning of OAI-PMH to understand how it can take advantage of the NS-M and INS-M and how it can be extended to cope with the exchange of hierarchical structures. OAI-PMH is so widely diffuse in the field of DL that we can assume the reader is familiar with its underlying functioning. The main feature of OAI-PMH we exploit is selective harvesting; this is based on the concept of $O A I$-set, which enables logical data partitioning by defining groups of records. Selective harvesting is the procedure that permits the harvesting only of metadata owned by a specified OAI-set. In OAI-PMH a set is defined by three components: setSpec which is mandatory and a unique identifier for the set within the repository, setName which is a mandatory short human-readable string naming the set, and setDesc which may hold community-specific XML-encoded data about the set. 
OAI-set organization may be hierarchical, where hierarchy is expressed in the setSpec field by the use of a colon [:] separated list indicating the path from the root of the set hierarchy to the respective node. For example if we define an OAI-set whose setSpec is " $A$ ", its subset " $B$ " would have " $A: B$ " as setSpec. In this case " $\mathrm{B}$ " is a proper subset of "A": $B \subset A$. When a repository defines a set organization it must include set membership information in the headers of the records returned to the harvester requests. Harvesting from a set which has sub-sets will cause the repository to return the records in the specified set and recursively to return the records from all the sub-sets. In our example, if we harvest set $\mathrm{A}$, we also obtain the records in sub-set $\mathrm{B}[13$.

In OAI-PMH it is possible to define an OAI-set organization based on the NS-M or INS-M. This means that we can treat the OAI-sets as a Nested Set Family (NS-F) or as an Inverse Nested Set Family (INS-F). The inclusion order between the OAI-sets is given by its identifier which is a <setspec > value. This <setspec> value is also added in the header of every record belonging to an OAI-set. In the following we describe how it is possible to create a Nested Set family of OAI-Set and afterward how the same thing can be done with an Inverse Nested Set family.

Let $\mathcal{O}$ be a Nested Set family and let $I$ be the set of the <setspec> values where $i \in I=\left\{s_{0}: s_{1}: \ldots: s_{j}\right\}$ means that $\exists O_{j} \in\left\{O_{i}\right\}_{i \in I} \mid O_{j} \subset \ldots \subset$ $O_{1} \subset O_{0}$. Every $O_{i} \in\left\{O_{i}\right\}_{i \in I}$ is an OAI-set uniquely identified by a <setspec> value in $I$. The <setspec> values for the $O_{k} \in\left\{O_{i}\right\}_{i \in I}$ are settled in such a way to maintain the inclusion order between the sets. If an $O_{k}$ has no superset its setspec value is composed only by a single value ( $\langle$ setspec $\rangle s_{k}</$ setspec $\rangle$ ). Instead if a set $O_{h}$ has supersets, e.g. $O_{a}$ and $O_{b}$ where $O_{b} \subset O_{a}$, its setspec value must be the combination of the name of its supersets and itself separated by the colon [:] (e.g. 〈setspec $>s_{a}: s_{b}: s_{h}</$ setspec $>$ ). Furthermore, let $R=$ $\left\{r_{0}, \ldots, r_{n}\right\}$ be a set of records, then each $r_{i} \in O_{j}$ must contain the setspec of $O_{j}$ in its header.

Throughout $\left\{O_{i}\right\}_{i \in I}$ it is possible to represent a hierarchical data structure, such as a tree, in OAI-PMH providing a granularity access to the items in the hierarchy and at the same time enabling the exchange of a single part of the hierarchy with the possibility of reconstructing the whole hierarchy whenever it is necessary. The next section presents a concrete use case of the NS-F mapping a tree data structure enabling its representation and exchange by means of OAIPMH; a visual idea of this procedure can be seen in Fig. 4.

In the same way we can apply the INS-M to OAI-PMH; Let $\mathcal{U}$ be an Inverse Nested Set family and let $J$ be the set of the <setspec> values where $j \in$ $J=\left\{s_{0}: s_{1}: \ldots: s_{k}\right\}$ means that $\exists U_{k} \in\left\{U_{j}\right\}_{j \in J}=U_{k} \subset \ldots \subset U_{1} \subset$ $U_{0}$. In $\left\{U_{j}\right\}_{j \in J}$ differently that in $\left\{O_{i}\right\}_{i \in I}$ the following case may happen: Let $U_{i}, U_{k}, U_{w} \in\left\{U_{j}\right\}_{j \in J}$ then it is possible that $U_{w} \subset U_{i}$ and $U_{w} \subset U_{k}$ but either $U_{i} \nsubseteq U_{k}$ and $U_{k} \nsubseteq U_{i}$. If we consider $\left\{U_{j}\right\}_{j \in J}$ composed only of $U_{i}, U_{k}$ and $U_{w}$, the identifier of $U_{i}$ is $\left\langle\right.$ setspec $>s_{i}</$ setspec $>$ and the identifier of $U_{k}$ is $\left\langle\right.$ setspec $>s_{k}</$ setspec $>$. Instead, the identifier of $U_{w}$ must be $\left\langle\right.$ setspec $>s_{i}$ : $s_{w}</$ setspec $>$ and $\left\langle\right.$ setspec $>s_{k}: s_{w}</$ setspec $>$ at the same time; this means 


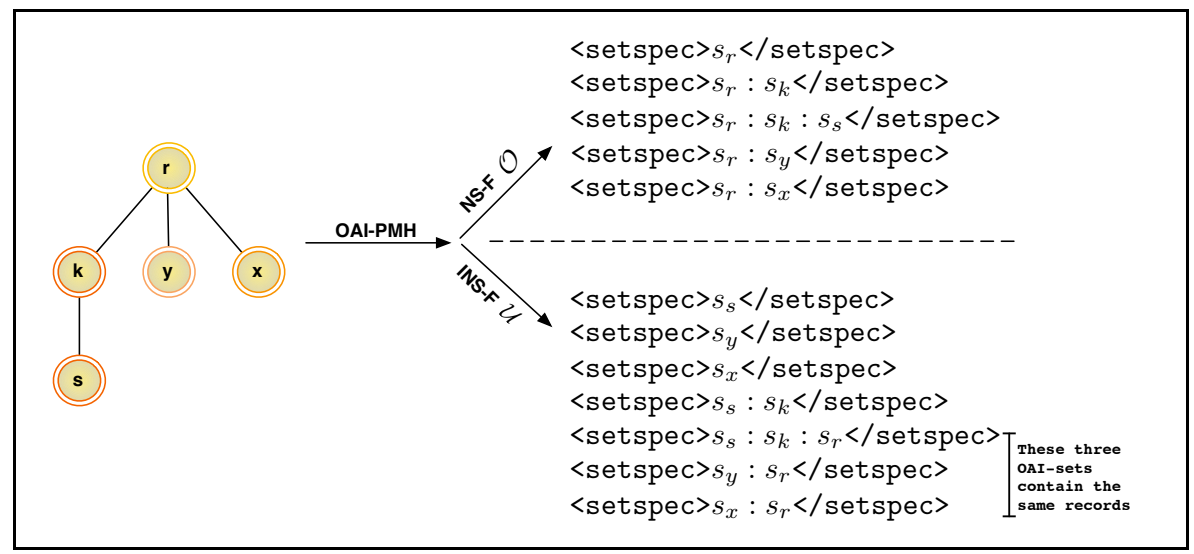

Fig. 3. The setspec values of the OAI-sets belonging to the NS-F $\left\{O_{i}\right\}_{i \in I}$ and the INS-F $\left\{U_{j}\right\}_{j \in J}$ obtained from a sample tree

that in $\left\{U_{j}\right\}_{j \in J}$ there are two distinct OAI-sets, one identified by $\left\langle\right.$ setspec $>s_{i}$ : $s_{w}</$ setspec $>$ and the other identified by $\left\langle\right.$ setspec $>s_{k}: s_{w}</$ setspec $>$. This is due to the fact that the intersection between OAI-sets in OAI-PMH is not defined set-theoretically; indeed, the only way to get an intersection of two OAI-sets is enumerating the records. This means that we can know if an OAI-record belongs to two or more sets just by seeing whether there are two or more <setspec> entries in the header of the record. In this case the records belonging to $U_{w}$ will contain two <setspec > entries in their header: <setspec $>s_{i}: s_{w}</$ setspec $>$ and $\left\langle\right.$ setspec $>s_{k}: s_{w}</$ setspec $>$; note that only the $\langle$ setspec $>$ value is duplicated and not the records themselves.

In Figure 3 we can see how a sample tree can be represented in OAI-PMH exploiting the OAI-sets organization. In the upper part we reported the <setspec> values of the OAI-sets organized in a NS-F $\left\{O_{i}\right\}_{i \in I}$, instead in the lower part we reported the <setspec > values of the OAI-sets organized in INS-F $\left\{U_{j}\right\}_{j \in J}$.

With this view of OAI-PMH we can set a hierarchical structure of items as a well-defined nested set organization that maintains the relationships between the items just as a tree data structure does and moreover we can exploit the flexibility of the sets exchanging a specific subset while maintaining the integrity of the data. Indeed, in the header of the items there is the set membership information which, if necessary, enables the reconstruction of the hierarchy or part of it. Throughout the NS-M and INS-M it is possible to handle hierarchical structures in OAI-PMH simply by exploiting the inner functionalities of the protocol; indeed, no change of OAI-PMH is required to cope with the presented set data models.

The choice between NS-M and INS-M is based on the application context: NS-M fosters the reconstruction of the lower levels of a hierarchy starting from a node, vice versa INS-M fosters the reconstruction of the upper levels. This difference between the models should become clearer if we consider a relevant example of how OAI-PMH and the presented set data models can be successfully used to overcome well-known problems in data exchange. 


\subsection{The Set Data Models and OAI-PMH Applied to the Archives}

This subsection describes how we can exchange archival metadata in a distributed environment and it is a continuation of the work presented in [5. A brief introduction regarding the archive peculiarities is worthwhile for a better understanding of the proposed solutions. An archive is a complex cultural organization which is not simply constituted by a series of objects that have been accumulated and filed with the passing of time. Archives have to keep the context in which their documents have been created and the network of relationships among them in order to preserve their informative content and provide understandable and useful information over time. The context and the relationships between the documents are preserved thanks to the strongly hierarchical organization of the documents inside the archive. Indeed, an archive is divided by fonds and then by sub-fonds and then by series and then by sub-series and so on; at every level we can find documents belonging to a particular division of the archive or documents describing the nature of the considered level of the archive (e.g. a fond, a sub-fonds, etc.). The union of all these documents, the relationships and the context information permits the full informational power of the archival documents to be maintained.

In the digital environment an archive and its components are described by the use of metadata; these need to be able to express and maintain such structure and relationships. The standard format of metadata for representing the complex hierarchical structure of the archive is Encoded Archival Description (EAD) [9], which reflects the archival structure and holds relations between documents in the archive. On the other hand to maintain all this information an EAD file turns out to be a very large XML file with a deep hierarchical internal structure. Thus, accessing, searching and sharing individual items in the EAD might be difficult without taking into consideration the whole hierarchy. On the other hand, users are often interested in the information described at the item level, which is typically buried very deeply in the hierarchy and might be difficult to reach [11]. These issues can be overcome by describing the hierarchical organization of an archive as a family of sets in the NS-M, where the documents belonging to a specific division of the archive become metadata belonging to a specific set.

In Fig. 4 we can see two approaches to representing the archival organization and documents. The first approach is the EAD-like one in which the whole archive is mapped inside a unique XML file which is potentially very large and deeply hierarchical. All information about fonds, sub-fonds or series as well as the documents belonging to a specific archival division are mapped into several XML elements in the same XML file. With this approach we cannot exchange precise metadata through OAI-PMH, rather we have to exchange the whole archive. At the same time it is not possible to access a specific piece of information without accessing the whole hierarchy [10.

The second approach is based on the NS-M. The archival hierarchy is mapped into a family $\Phi$ that for theorem 1 is a NS-F. In $\Phi$ the documents are represented as items belonging to the opportune set. In this way the context information and the relationships between the documents are preserved thanks to the nested set 


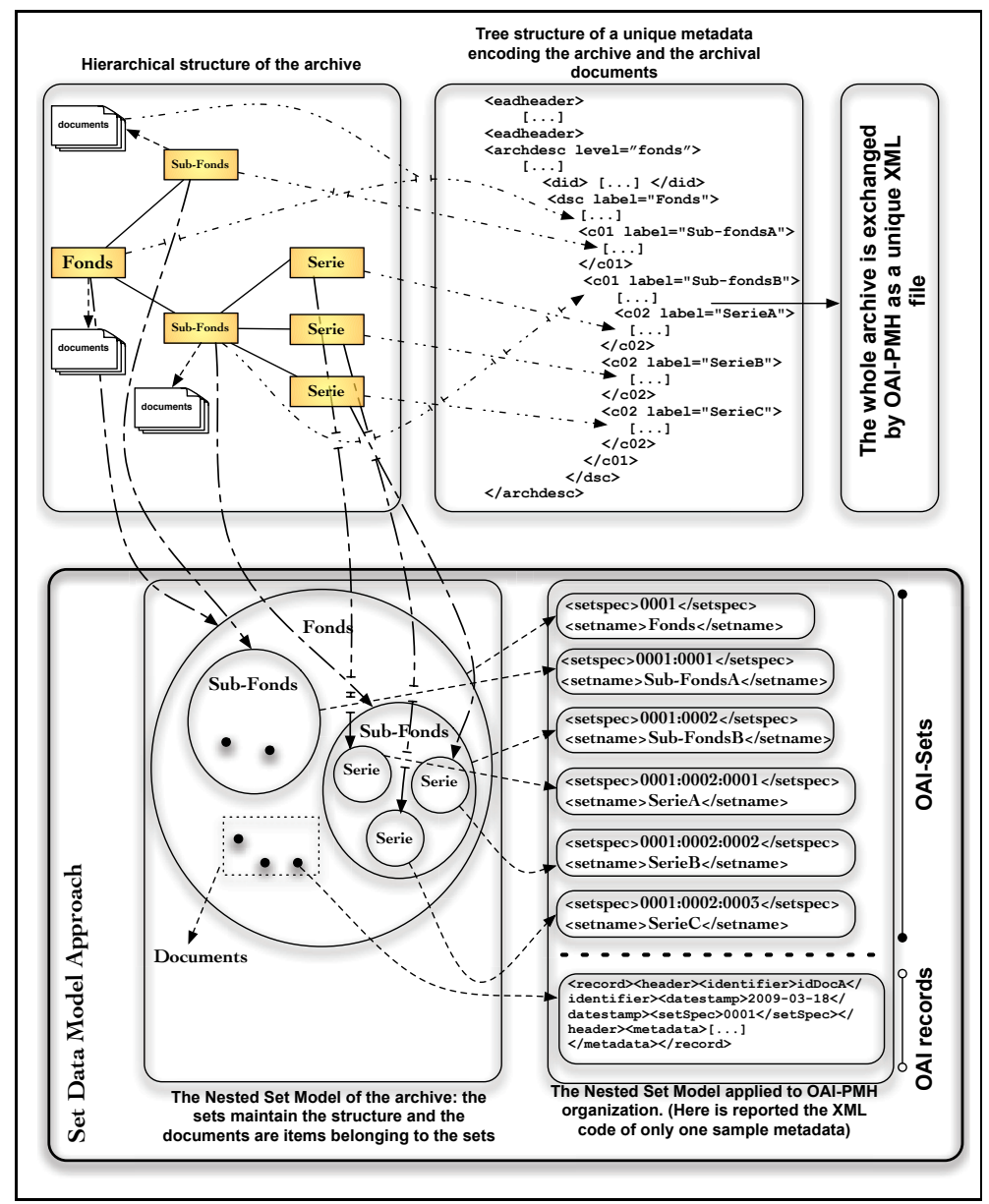

Fig. 4. The hierarchical structure of an archive mapped into a metadata with a tree data structure, the alternative mapping in the NS-M and in OAI-PMH

organization and at the same time they are not bound to a rigid structure. Then, $\Phi$ is represented in OAI-PMH throughout the family $\left\{O_{i}\right\}_{i \in I}$ of OAI-sets obtained setting of the <setspec> values as described in the previous subsection. For instance, the set obtained from the root has a "0001" identifier, the set mapped from the children of the root are identified by "0001:0001", "0001:0002" and so on. Thus, from the identifier of an OAI-set we can reconstruct the hierarchy through the ancestors to the root. By means of OAI-PMH it is possible to exchange a specific part of the archive while at the same time maintaining the relationships with the other parts of it. The NS-M fosters the reconstruction of the lower levels of a hierarchy; thus, with the couple NS-M and OAI-PMH applied to the archive, if a harvester asks for an OAI-set representing for instance a sub-fond it recursively obtains all the OAI-subsets and items in the subtree rooted in the selected sub-fonds. 
This approach can also be applied with the INS-M mapping the archival hierarchy into a INS-F $\left\{U_{j}\right\}_{J \in J}$ following the procedure illustrated in the previous section. In this case there is a big difference in the harvesting procedure; indeed, if a harvester asks for an OAI-set representing for instance an archival series it recursively obtains all the OAI-subsets and records in the path from the archival series to the principal fond that is the root of the archival tree. The choice between a NS-M or INS-M should be done on the basis of the application context. In the archival context the application of the INS-M would be more significant than the NS-M. Indeed, often the information required by a user stored in the external nodes of the archival tree 11. If we represent the archival tree by means of the INS-F, when a harvester requires an external node of the tree it will receive all the archival information contained in the nodes up to the root of the tree. This means that a Service Provider can offer a potential user the required information stored in the external node and also all the information stored in its ancestors nodes. This information is very useful for inferring the context of an archival metadata which is contained in the required external node; indeed, the ancestor nodes represent and contain the information related to the series, sub-fonds and fonds in which the archival metadata are classified. The INS-M fosters the reconstruction of the upper levels of a hierarchy that in the archival case often contain contextual information which permit the relationships of the archival documents to be inferred with the other documents in the archive and with the production and preservation environment.

\section{Conclusions}

We have discussed the relevance of the hierarchical structures in computer science with a specific examination of the DLSs. We have presented the tree data structure and highlighted the more relevant aspects to our treatment of hierarchical structures. We have also presented the NESTOR framework defining two set-theoretical data models called Nested Set Model and Inverse Nested Set Model as alternatives of the tree data structure. Furthermore, we have shown how a tree can be mapped in one model or the other. These models maintain the peculiarities of the tree with the flexibility and accessibility of sets. We have shown how the protocol OAI-PMH can be extended by exploiting the NS-M or the INS-M. Lastly we have presented a significant application of the presented set data models in conjunction with OAI-PMH represented by the archives. Indeed, we have shown how the hierarchical archive organization can be represented and exchanged in OAI-PMH and thus between different DLSs in a distributed environment.

\section{Acknowledgments}

The authors wish to thank Maristella Agosti for her support and collaboration to bring forth this work. The work reported has been supported by a grant from the Italian Veneto Region. The study is also partially supported by the TELplus 
Targeted Project for Digital Libraries, as part of the eContentplus Program of the European Commission (Contract ECP-2006-DILI- 510003).

\section{References}

1. Collins Dictionary of The English Language. William Collins Sons \& Co.Ltd (1979)

2. Anderson, K.W., Hall, D.W.: Sets, Sequences, and Mappings: The Basic Concepts of Analysis. John Wiley \& Sons, Inc., New York (1963)

3. Celko, J.: Joe Celko's SQL for Smarties: Advanced SQL Programming. Morgan Kaufmann, San Francisco (2000)

4. Davey, B.A., Priestley, H.A.: Introduction to Lattices and Order, 2nd edn. Cambridge University Press, Cambridge (2002)

5. Ferro, N., Silvello, G.: A Methodology for Sharing Archival Descriptive Metadata in a Distributed Environment. In: Christensen-Dalsgaard, B., Castelli, D., Ammitzbøll Jurik, B., Lippincott, J. (eds.) ECDL 2008. LNCS, vol. 5173, pp. 268-279. Springer, Heidelberg (2008)

6. Halmos, P.R.: Naive Set Theory. D. Van Nostrand Company, Inc., New York (1960)

7. Jech, T.: Set Theory - The Third Millenium Edition. Springer, Heidelberg (2003)

8. Knuth, D.E.: The Art of Computer Programming, 3rd edn., vol. 1. Addison Wesley, Reading (1997)

9. Pitti, D.V.: Encoded Archival Description. An Introduction and Overview. D-Lib Magazine 5(11) (1999)

10. Prom, C.J., Rishel, C.A., Schwartz, S.W., Fox, K.J.: A Unified Platform for Archival Description and Access. In: Rasmussen, E.M., Larson, R.R., Toms, E., Sugimoto, S. (eds.) Proc. 7th ACM/IEEE Joint Conference on Digital Libraries (JCDL 2007), pp. 157-166. ACM Press, New York (2007)

11. Shreeves, S.L., Kaczmarek, J.S., Cole, T.W.: Harvesting Cultural Heritage Metadata Using the OAI Protocol. Library Hi Tech. 21(2), 159-169 (2003)

12. Van de Sompel, H., Lagoze, C., Nelson, M., Warner, S.: Implementation Guidelines for the Open Archive Initiative Protocol for Metadata Harvesting. Technical report, Open Archive Initiative (2002)

13. Van de Sompel, H., Lagoze, C., Nelson, M., Warner, S.: Implementation Guidelines for the Open Archive Initiative Protocol for Metadata Harvesting - Guidelines for Harvester Implementers. Technical report, Open Archive Initiative, p. 6 (2002) 